\title{
The Quality Prediction of Fiber-Optic Gyroscope Based on the Grey Theory and BP Neural Network
}

\author{
$\mathrm{Na} \mathrm{Ji}$ \\ Shanghai Key Laboratory of Mechanical Automation and \\ Robotics, School of Mechatronic Engineering and \\ Automation \\ Shanghai University \\ Shanghai, China \\ 80709412@qq.com
}

\author{
Hongxia Cai* \\ Shanghai Key Laboratory of Mechanical Automation and \\ Robotics, School of Mechatronic Engineering and \\ Automation \\ Shanghai University \\ Shanghai, China \\ hxcai@staff.shu.edu.cn
}

\begin{abstract}
Fiber-optic gyroscope has become a new generation of leading device of inertial measurement system. Fiber-optic gyroscope is small batch product with high precision and high cost. Its development process has the characteristics of heavy testing work, complex data analysis, poor stability so that its product quality is hard to control. Therefore, we use the grey theory combined with BP neural network to build the model for the quality prediction of fiber-optic gyroscope. As the fiber-optic gyroscope is small batch product and its quality is influenced by multiple factors, we add "The Sample Selector" and optimize the results in the traditional grey prediction method. "The Sample Selector" is used to process the relevant historical data of similar fiber-optic gyroscope. The grey model is used to predict the quality of the fiber-optic gyroscope. Furthermore, we use BP neural network to correct residual error to improve the accuracy of prediction. We verified that the method has a high accuracy rate and meets the production demands. According to the prediction, it can take timely measures to control the product quality in advance.
\end{abstract}

Keywords-Fiber-optic gyroscope; grey theory; BP neural network; quality prediction

\section{INTRODUCTION}

Fiber-optic gyroscope has the unique advantages in the aerospace, aviation, navigation, weapon and industry areas as a new generation of angular velocity sensitive device [1]. At present it has been widely used as guided torpedo, fiber optical guided missile, subsurface exploration, ground vehicle position and orientation, airborne inertial navigation system, etc. especially in the military field. Fiber-optic gyroscope has made a very wide range application which is unmatched by any other gyroscope. According to the fiber-optic gyroscope market report of Business Communications Company, the globe fiber-optic gyroscope market value has been keeping the average annual compound growth rate $4.1 \%$ in recent years. According to the research report of Global Industry Analysts, the Asia-Pacific region is the global main market of fiber-optic gyroscope, in which the United States and China account for $39 \%$ of the total. Therefore, many countries strengthen the research of fiber-optic gyroscope. Fiber-optic gyroscope is small batch product with high precision and high cost. As its development process has heavy testing work, complex data analysis and poor stability, product quality is difficult to control. The test work accounts for $30 \%$ of the whole development process for fiber-optic gyroscope. On the basis of a large number of accumulated test data, we should analyze the main parameters of fiber-optic gyroscope data to forecast the quality of fiber-optic gyroscope so that we could control the product quality in advance. That will be an effective way in improving the quality of fiber-optic gyroscope [2].

Currently there are lots of forecasting methods including expert forecasting method (such as Brainstorming, Delphi Technique, etc.), trend extrapolation, exponential smoothing, analogy method, regression analysis, the inputs and outputs analysis, growth curve method, artificial neural network models [3]. Wu and Shieh used a Markov chain model in quality function deployment to analyze customer requirements [4]. Deng et al. hybridized the genetic algorithm and neural network for process parameters optimization [5]. Wang et al. put forward grey system theory based prediction for topic trend on Internet [6]. These algorithms have been applied to the various industry product quality predictions. Based on the grey sequence method and the grey relational space analysis, the grey theory can achieve the analysis, evaluation, modeling, forecasting, decision-making, control and optimization. David et al. introduced the BP algorithm into the traditional quality control chart, training and test, and it is feasible that the artificial neural network can predict abnormal situation in quality control [7]. Wang et al. set the process parameters intelligently for layered manufacturing by developing the BP neural network [8]. Sanjukta and William present a multi-variable model which combined the recursive neural network and the enhanced evolutionary algorithm to monitor and correct dynamic process [9]. It is effective in controlling the quality influenced by multi-variable. Compared with other forecasting methods, the grey theory has the characteristics such as: it is feasible and sample data are small, with four data enough in theory; Samples do not need to have a regular distribution; The computing workload is small; The quantitative analysis and the qualitative analysis of the results would be consistent; It can be used in the recent, mid, and long-term prediction, and the accuracy of prediction is high [10]. Wei established an improved model combined the traditional GM $(1,1)$ and RBF neural network and use the advantages of both to build grey compensating RBF neural 
network forecasting mode, which can select optimal background value and dynamic identifying parameters [11]. However, the grey theory has its own limitation: the results sometimes are messy; it is not as accurate as the regression analysis method in the situation with large number of sample data. Therefore many scholars put forward the corresponding improved algorithms when they use the grey theory. As the convolution transform can improve the transformation sequence smoothness and the mechanism of accumulation generation, Chen improved the sequence generation of grey model and GM $(1,1)$ model and established the grey model GM (1, 1, t) with linear time [12]. Wang and Zhang established the grey combination prediction model based on ant colony algorithm [13]. Wan et al. established forecasting model for the material consumption based on the grey theory and neural network model, meanwhile he presented a new kind of broadly combination forecast model in which he improved the function for the generalized weighted and proposed the way to estimate weighted coefficient of the parameter [14].

Based on the analysis of the above, the grey theory is an effective method to predict with small sample data. With the help of the grey theory, the behavior and the evolution of the uncertain system could be correctly described and effectively monitored. As fiber-optic gyroscope is small-batch ordered product, it is suitable to use the grey theory to predict the product quality [15-17]. However in order to further improve the accuracy of the result, we add "The Sample Selector" and optimize the result with BP neural network based on the traditional grey prediction SCGM $(1,1)$ model [18]. The sample processor is to analysis relevant historical data of the fiber-optic gyroscope. Combining with the BP neural network, it corrects the data residual from the grey model in the results optimizer, because BP neural network can avoid the distorting phenomenon which is generated from the sequence accumulation offset. In this paper, we predict the product quality of fiber-optic gyroscope by the combination model of the grey theory and BP neural network. We verify that the method has a high accuracy rate and meets the production demands.

\section{PROBLEM DESCRIPTION}

Fiber-optic gyroscope is a new type sensor with no rotation of quality which can measure angular velocity and rotation angle of the object relative to the inertial space. The applications of fiber-optic gyroscope in aviation, airborne systems and military technology are increasing, especially gain extensive attention of the army. Taking America, Japan, France as the main body, the fiber-optic gyroscope research work has made great progress. It came into commercial as early as 1990s. The precision of modern fiber-optic gyroscope has amounted to $0.0001 \% \mathrm{~h}$. The prediction and control of quality is becoming more and more important of fiber-optic gyroscope enterprise production process. We can realize advanced control product quality, scientific and reasonable planning and effectively ensure the quality of the product by quality forecast. There are many important testing indexes, such as the scale factor, zero bias, threshold, and other indicators which are reflected the quality of fiber-optic gyroscope [19]. The scale factor is the proportion of the maximum output to the maximum deviation which is the fiber-optic gyroscope output relates to the least square method fitting line in the scope of the inputs angular velocity. Due to the working point of fiber-optic gyroscope varies with the change of the angular velocity, the scale factor will also change. The greater the inputs angular velocity is, the greater the nonlinearity is. So the scale factor is an important index to predict the quality of the fiber-optic gyroscope [20]. This article proposes the grey theory combined with BP neural network method to forecast the product quality and use the sample data of the scale factor in the fiber-optic gyroscope testing indexes for validation. The result proves the feasibility of the algorithm.

\section{Algorithm OF THE QuALITy PREDICTION}

The flow chat of the product quality prediction model based on the grey theory and BP neural network is shown in Fig. 1. It includes the sample selection and processing, quality prediction (the grey theory), the optimization of result (BP neural network) and trace analysis.

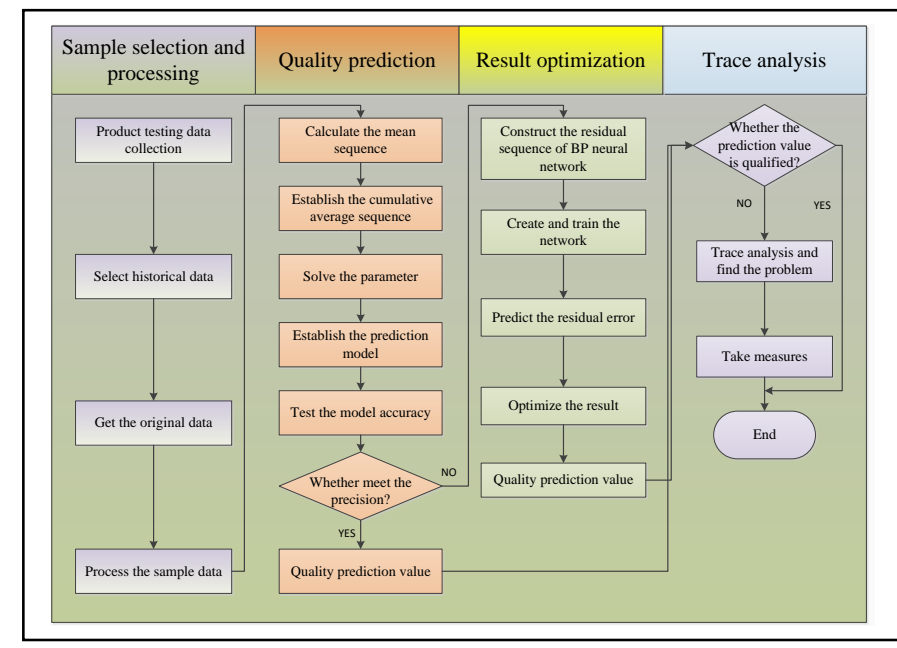

Fig. 1. Flow chat of the product quality prediction.

\section{A. Sample selection and processing}

The main function of sample selection and processing is to dig the historical data of the fiber-optic gyroscope in order to provide more accurate inputs for $\operatorname{SCGM}(1,1)$ model. There are two ways to use the original sample data: one is the direct use of the original sample data and the other is selecting the optimal sample data in some way. For example, the optimal sample data is chosen by the grey fuzzy clustering evaluation method [21]. However, they don't pay attention to the fact that different sample data have distinct different effect. In this paper, considering that the latest data will be the most valuable to predict, so we choose the latest product data from the history samples as new forecast data to constitute a new prediction sample. Assuming that the historical sample data of fiber-optic gyroscope test scale factor are as follows:

$$
X_{0}=\left\{x^{(0)}(1), x^{(0)}(2), \cdots, x^{(0)}(n)\right\}
$$

Among this: $\mathrm{n}$ is the number of sample size. 
New prediction sample data are:

$$
X_{1}=\left\{x^{(0)}(1), x^{(0)}(2), \cdots, x^{(0)}(n), x^{(0)}(s)\right\}
$$

Among this, $s$ is the number of new prediction sample size, we assume $\mathrm{s}=\mathrm{n}+1$ and new prediction sample are:

$$
X_{1}=\left\{x^{(0)}(1), x^{(0)}(2), \cdots, x^{(0)}(n), x^{(0)}(n+1)\right\}
$$

\section{B. Grey theory model SCGM $(1,1)$}

GM $(1,1)$ and SCGM $(1,1)$ are common grey forecasting models [22-23]. GM $(1,1)$ model is relatively simple and it is more commonly used. However, Yao et al.2012 presented that the GM $(1,1)$ model is not feasible for the situation with poor information and the $\operatorname{SCGM}(1,1)$ has more advantages to predict in the uncertain situation with poor information and multiple-factor for it has more rigorous logic structure.

Considering the quality is influenced by many factors, we choose SCGM $(1,1)$ model. The steps of the grey theory SCGM $(1,1)$ model are as follows:

\section{1) Determine the forecast sample}

The sample $\mathrm{X}_{1}$ which was processed by sample processor will be the $\operatorname{SCGM}(1,1)$ model of the original sample $X^{(0)}$.

$$
\begin{aligned}
& X^{(0)}=X_{1}= \\
& \left.\left.\left\{{ }^{(0}\right)(), 1^{0}, X() 2 ; \cdot, \stackrel{(0}{X}\right)(n), x^{(0)}(n+1)\right\}
\end{aligned}
$$

2) Calculate the average

$$
\begin{aligned}
& x^{(0)}(t)=0.5\left(x^{(0)}(t)+x^{(0)}(t-1)\right), \\
& t=2,3, \cdots, n+1
\end{aligned}
$$

And:

$$
\bar{X}^{(0)}(t)=\left\{x^{-(0)}(2), x^{-(0)}(3), \cdots, x^{-(0)}(n), x^{-(0)}(n+1)\right\}
$$

\section{3) Calculate the cumulative average sequence}

$$
\bar{x}^{(1)}(k)=\sum_{t=2}^{k} \bar{x}^{(0)}(t), k=2,3, \cdots, n, n+1
$$

We can obtain the cumulative average sequence:

$$
\bar{X}^{(1)}(k)=\left\{x^{-(1)}(2), x^{-(1)}(3), \cdots, x^{(1)}(n), x^{(1)}(n+1)\right\}(8)
$$

\section{4) Determine parameters}

Make $s(f)$ as a nonhomogeneous discrete exponential function. Assuming that $\mathrm{f}_{\mathrm{r}} \in \mathrm{s}(\mathrm{f})$ is a certain function in time domain and considering the general form of generalized energy system. We make

$$
\begin{aligned}
& f_{r}(k)=b e^{a(k-1)}-c \\
& a, b, c \in R, k=1,2,3, \cdots, n
\end{aligned}
$$

If $\left\{\overline{\mathrm{X}}^{(1)}(\mathrm{k})\right\}$ is associated with satisfaction trend of nonhomogeneous discrete exponential function we use the least square method. Parameters $\mathrm{a}$ and $\mathrm{b}$ are confirmed as follows:

$$
a=\ln \frac{\sum_{t=3}^{n+1} \bar{x}^{(0)}(t-1)^{-(0)}(t)}{\sum_{t=3}^{n+1}\left(\bar{x}^{(0)}(t-1)\right)^{2}}
$$

$$
\begin{gathered}
b=\frac{n \sum_{k=2}^{n+1} e^{a(k-2)} x^{-(1)}(k)-\left(\sum_{k=2}^{n+1} e^{a(k-2)}\right)\left(\sum_{k=2}^{n+1}-(1)(k)\right)}{n \sum_{k=2}^{n+1} e^{2 a(k-2)}-\left(\sum_{k=2}^{n+1} e^{a(k-2))}\right.} \\
c=\frac{1}{n}\left|\sum_{k=2}^{n+1} e^{2 a(k-2)}-\sum_{k=2}^{n+1} \bar{X}^{(1)}(k-1)\right|
\end{gathered}
$$

5) Build up the forecast model

The forecast model is:

$$
\tilde{X}^{(0)}(t)=b\left(1-e^{-a}\right) e^{a(t-1)}
$$
is:

Then performance index of the forecasting data QuaConf

$$
\text { QuaConf }=\tilde{X}^{(0)}(n+2)=b\left(1-e^{-a}\right) e^{a(t-1)}
$$

6) Verify the model accuracy

$$
\begin{aligned}
q^{(0)}(t) & =x^{(0)}(t)-\tilde{X}^{(0)}(t) \\
e(t) & =q^{(0)}(t) / X_{X}^{(0)}(t)
\end{aligned}
$$

$\mathrm{q}^{(0)}(\mathrm{t})$ is residual in (15) and $\mathrm{e}(\mathrm{t})$ is the relative error in (16) Normally we think $\mathrm{e}(\mathrm{t}) \leq 5 \%$ is a good result. Or we use posterior-variance-test. The method is shown below:

Get the average of $x^{(0)}(t)$ :

$$
\bar{x}=\frac{1}{n+1} \sum_{t=1}^{n+1} x^{(0)}(t)
$$


Get the variance of $x^{(0)}(t)$ :

$$
S_{1}^{2}=\frac{1}{n+1} \sum_{t=1}^{n+1}\left(x^{(0)}(t)-\bar{x}\right)^{2}
$$

Get the average of the residuals $\mathrm{q}^{(0)}(\mathrm{t})$ :

$$
\bar{q}=\frac{1}{n+1} \sum_{t=1}^{n+1} q^{(0)}(t)
$$

Get the variance of $q^{(0)}(t)$ :

$$
S_{2}^{2}=\frac{1}{n+1} \sum_{t=1}^{n+1}\left(q^{(0)}(t)-\bar{q}\right)^{2}
$$

The ratio of the mean square $\mathrm{C}$ is:

$$
C=S_{2} / S_{1}
$$

The probability of small error $\mathrm{P}$ is:

$$
P=P\left\{q^{(0)}(t)-\bar{q} \mid<0.6745 S_{1}\right\}
$$

The small the error ratio of mean square is, the better the result is. If $C$ is smaller, then $S_{1}$ is bigger or $S_{2}$ is smaller. The historical test scale factor data variance is bigger and the degree of discrete is greater if $S_{1}$ is bigger. The residual variance is smaller and the degree of discrete is smaller if $S_{2}$ is smaller. $\mathrm{C}$ is small that means although the historical test scale factor data are discrete, the difference between the predicted and actual values is not too discrete.

The bigger the probability of small error is, the better result we get. If $\mathrm{P}$ is bigger, it means the difference between the residual and the average residual is pretty less than the given value $0.6754 \mathrm{~S}_{1}$. According to the index of $\mathrm{C}$ and $\mathrm{P}$, we can comprehensively assess the accuracy of scale factor, as shown in Table I.

If the result is unsatisfied, we need to use the residual sequence to establish the BP neural network model to optimize the result.

TABLE I. CORRESPONDING RELATIONS BETWEEN IMPACT LEVEL AND SCORE

\begin{tabular}{|c|c|c|}
\hline Precision Grade & P & C \\
\hline Level1 & $>0.95$ & $<0.35$ \\
\hline Level2 & $>0.80$ & $<0.50$ \\
\hline Level3 & $>0.70$ & $<0.65$ \\
\hline Level4 & $\leq 0.70$ & $\geq 0.80$ \\
\hline
\end{tabular}

\section{Optimization Model}

The optimization steps based on the improved BP neural network is as follows:

1) Construct the residual sequence $q^{(0)}(t)$ of the $B P$ neural network model

The residual error is the difference of sample data $x^{(0)}(t)$ before time $\mathrm{t}$ subtract the predicted value from $\operatorname{SCGM}(1,1)$. If the forecast order is $n$, we use $\mathrm{q}^{(0)}(\mathrm{t}-1), \mathrm{q}^{(0)}(\mathrm{t}-2), \cdots, \mathrm{q}^{(0)}(\mathrm{t}-\mathrm{n})$ to calculate the prediction result in time and as the training sample inputs into neural network (inputs matrix). The $\mathrm{q}^{(0)}(\mathrm{t})$ is the expectations of network training (outputs matrix).

\section{2) Normalize sample}

Normalize the sample data to $[1,1]$ and the formula is as follows:

$$
y=\frac{\left(y_{\max }-y_{\min }\right)\left(x_{i}-x_{\min }\right)}{x_{\max }-x_{\min }}+y_{\text {min }}
$$

$x_{i}$ is the original data. $y_{\min }$ and $y_{\max }$ are the scope of naturalization and $[-1,1]$ is the default value.

\section{3) Sort data}

The $20 \%$ inputs data are used as test data and the $20 \%$ data are used as change data; others are used as normal inputs to train. We extract the above three groups of data randomly.

\section{4) Create and train the network}

According to the following method to determine the number of hidden layer nodes. Assume that input layer of BP neural network has $\mathrm{m}$ neurons, output layer has $\mathrm{n}$ neurons, hidden layer has s nodes, and then we can get the (24):

$$
s=\sqrt{m+n}+a
$$

$\mathrm{a}$ is constant between one to ten . We confirm the number of inputs and outputs characteristic parameters and the number of each layer of neurons, and choose a transfer function.

\section{5) Simulate network}

In order to further verify the network performance after training, the network should be simulated deeply. We use sample data for the trained network to simulate and it can be used as an effective tool for predicting residual sequence.

6) Determine and optimize the $q^{(0)}(t)$

The residual sequence which use training model of BP neural network to predict is $q^{(0)}(t)$ and we construct the new predicted value.

$$
X_{C}{ }^{(0)}=x_{c}^{(0)}(t)+q_{c}^{(0)}(t)
$$

$\mathrm{x}_{c}{ }^{(0)}(\mathrm{t})$ is the predicted value according to the $\operatorname{SCGM}(1,1)$ model. Its precision is higher than GM $(1,1)$, but there is still residual compared with actual value domain. We process the residual with BP artificial neural network, so we can get the more accurate residual error. According to the above analysis, $\mathrm{X}_{\mathrm{c}}{ }^{(0)}$ is the final predicted value. 


\section{CASE STUdy}

We investigate and survey data from a photoelectric technology co., LTD. We select batches of fiber-optic gyroscope test scale factor as the sample data. Nine consecutive batches of the $\mathrm{X}$ axis scale factor in normal temperature of the HI - L01 - I1 series fiber-optic gyroscope are shown in the following table.

TABLE II. HI - L01 - I1 SERIES FIBER-OPTIC GYROSCOPE SCALE FACTOR SAMPLE DATA

\begin{tabular}{|c|c|c|c|c|c|}
\hline Trial-manufacture & I & II & III & IV & V \\
\hline Scale Factor & 13957.4 & 14223.6 & 13215.9 & 9863.2 & 11459.0 \\
\cline { 1 - 5 } Trial-manufacture & VI & VII & VIII & IX & \\
\cline { 1 - 4 } Scale Factor & 11102.4 & 11102.4 & 9230.1 & 10612.7 & \\
\hline
\end{tabular}

Steps are as follows:

1) Process sample with the sample processor

The original data as scale factor of HI- L01-I1 series fiber-optic gyroscope are:

$$
X_{0}=\left\{\begin{array}{ccc}
13957.4 & 14223.6 & 13215.9 \\
9863.2 & 11459.0 & 11102.4 \\
10872.5 & 9230.1 & 10612.7
\end{array}\right\}
$$

There are nine data samples $(n=9)$. The latest batch data is IX. Increase the 10th data in the original historical samples and the latest data is 10612.7. The sample data are:

$$
X_{1}=\left\{\begin{array}{cccc}
13957.4 & 14223.6 & 13215.9 \\
9863.2 & 1459.0 & 11102.4 \\
10872.5 & 9230.1 & 10612.7 & 10612.7
\end{array}\right\}
$$

2) Use SCGM $(1,1)$ model to predict

Sample data are:

$$
X_{0}=X_{1}=\left\{\begin{array}{ccc}
14223.6 & 13215.9 & 9863.2 \\
11459.9 & 11102.4 & 10872.5 \\
9230.1 & 10612.7 & 10612.7
\end{array}\right\}
$$

The average series are calculated by (6):

$$
\bar{X}^{(0)}(t)=\left\{\begin{array}{ccc}
14090.5 & 13719.75 & 11539.55 \\
10661.1 & 11280.7 & 10987.45 \\
10051.3 & 9921.4 & 10612.7
\end{array}\right\}
$$

The samples generated by the average value accumulates according to (7) are:

$$
\bar{X}^{(1)}(t)=\left\{\begin{array}{ccc}
14090.5 & 27810.25 & 39349.8 \\
50010.9 & 61291.6 & 72279.05 \\
82330.35 & 92251.75 & 102864.45
\end{array}\right\}
$$

Parameters a and b are calculated by (10) and (11):

$$
\begin{aligned}
& a=-0.044168 \\
& b=-294342.31
\end{aligned}
$$

According to (13), the predicting model is:

$$
\begin{aligned}
& \tilde{X}^{(0)}(t)=b\left(1-e^{-a}\right) e^{a(t-1)} \\
& =-294342.31\left(1-e^{-0.044168}\right) e^{0.044168(t-1)}
\end{aligned}
$$

We use posterior-variance-test to verify.

According to the prediction model, predicting sequence is calculated.

$$
\widetilde{X}^{(0)}(t)=\left\{\begin{array}{ccc}
13773.86 & 13022.98 & 12313.02 \\
11641.78 & 11007.12 & 10407.06 \\
9839.72 & 9303.30 & 9267.30
\end{array}\right\}
$$

Then we get residual error sequence by (15).

$$
q^{(0)}(t)=\left\{\begin{array}{ccc}
665.49 & 1505.99 & 1047.78 \\
-1779.17 & 319.66 & 444.36 \\
647.96 & -526.84 & 1277.33
\end{array}\right\}
$$

The variance of the residual sequence is calculated by (19) and (20).

$$
S_{2}^{2}=908309.12
$$

Variance of the original value sequence is:

$$
S_{1}^{2}=2828229.97
$$

A posteriori difference ratio is calculated by (21).

$$
C=S_{2} / S_{1}=\frac{953.05}{1681.73}=0.57
$$

The little error frequency is calculated by (22).

$$
P=P\left\{q^{(0)}(t)-\bar{q} \mid<0.6745 S_{1}\right\}>0.95
$$

According to Table I, the model precision is Level 3. It is illustrated that SCGM $(1,1)$ prediction model is feasible and we can use it to predict product quality. But we should establish residual error correction model to further optimize the results. The forecast result of the grey theory model is:

$$
\text { QuaConf }(10)=b\left(1-e^{-a}\right) e^{a * 9}=11139.34
$$

3) Use the results corrector to optimize 
Establish residual error sequence $\mathrm{q}^{(0)}(\mathrm{t})$ of $\mathrm{BP}$ neural network model.

Input data is six groups and each group inputs three data.

$$
P=\left[\begin{array}{ccc}
665.49 & 1505.99 & 1047.78 \\
1505.99 & 1047.78 & -1779.17 \\
1047.78 & -1779.17 & 3199.66 \\
-1799.17 & 319.66 & 444.36 \\
319.66 & 444.36 & 674.96 \\
444.36 & 647.96 & -526.84
\end{array}\right]
$$

Output data is six groups and each group outputs one data.

$$
\begin{aligned}
& t=[-1779.17 ; 319.66 ; 444.36 ; 647.96 ; \\
& -526.84 ; 1277.3]
\end{aligned}
$$

4) Normalize the sample

Call the function of Matlab:

$$
\begin{aligned}
& {[\text { normInput, } p s]=\operatorname{map} \min \max (p)} \\
& {[\text { normInput, } t s]=\operatorname{map} \min \max (t)}
\end{aligned}
$$

\section{5) SortData}

By partitioning the training sample, we use dividevec() to extract three kinds of classification data randomly. The function call is as follows:

[trainSample, validateSamples, testSamples] $=$ divideveq $\left.\begin{array}{l}\text { normInput, normT arg et, } \\ \text { testPercent }\end{array}\right)$;

6) Create and train the network

Set the network parameters:

Node number of the first hidden layer is 20.

$$
\text { NodeNuml = 20; }
$$

Node number of the first hidden layer is 60 .

$$
\text { NodeNum2 = 60; }
$$

The dimension of the output is 1 .

$$
\text { TypeNum = 1; }
$$

Set the layers of transfer function:

$$
T F 1=T F 2=T F 3=' \text { transig' } ;
$$

TF3 is a transfer function of output layer.

Use the following code to create a network:

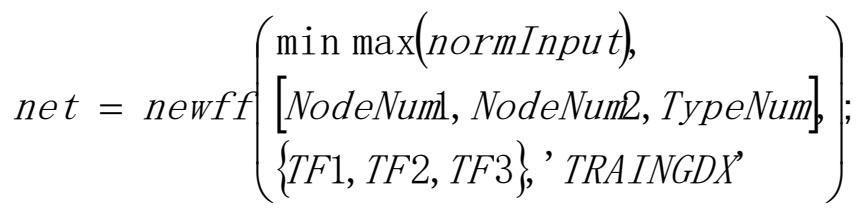

Use the following code to train a network:

$$
\begin{aligned}
& \text { net.trainParamepochs }=100000, \\
& \text { net.trainParamgoal }=1 e-4 ; \\
& \text { net.trainParam } 1 r=0.1 ;
\end{aligned}
$$

After network was created we begin to train the network and call the "train" function.

$$
[\text { net, } t r]=\text { train }\left(\begin{array}{l}
\text { net, trainSamples.p, } \\
\text { trainSamples.T, }[],[], \\
\text { validateSamples, testSamples }
\end{array}\right)
$$

\section{7) Simulate the network}

In order to validate the network performance after training, we the further simulate the network. We use the sample data validated to simulate the trained network. It is an effective tool for the residual sequence prediction. Fig. 2 illustrates the convergence process of training while it is close to target.

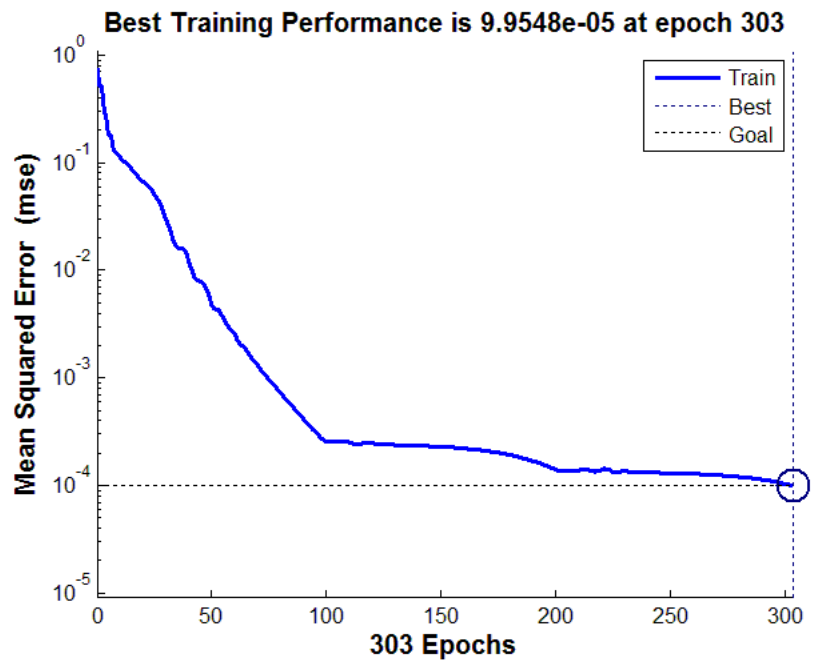

Fig. 2. BP neural network training.

The network test after trained is shown in Fig. 3. The "o" represents the actual value and "*" represents the predicted value. Fig. 4 shows the absolute error variation. According to Table III, the absolute prediction error is within 30 and relative error is less than $2 \%$. The precision is high and prediction error is small. So this network can be used as a residual error prediction. 


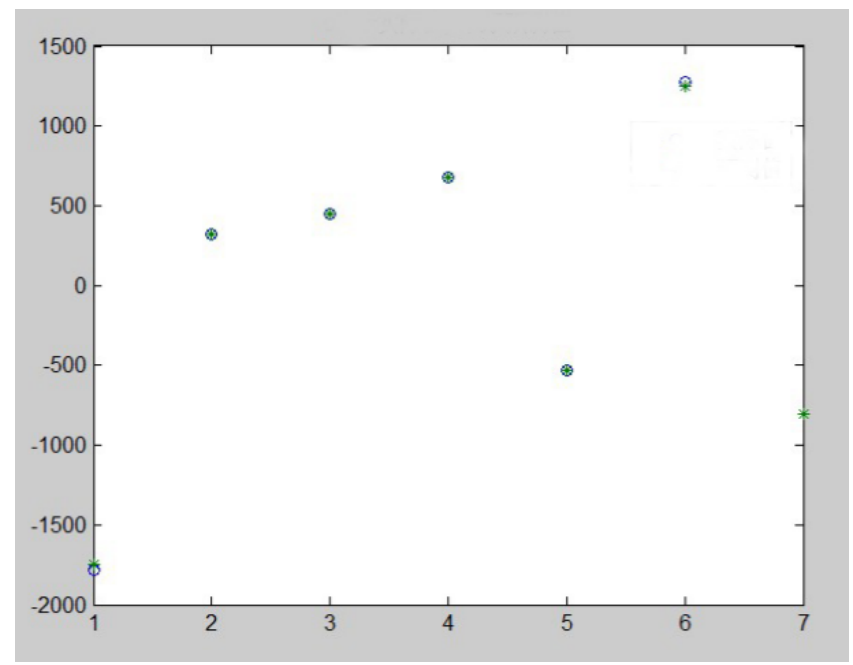

Fig. 3. Trained network prediction.

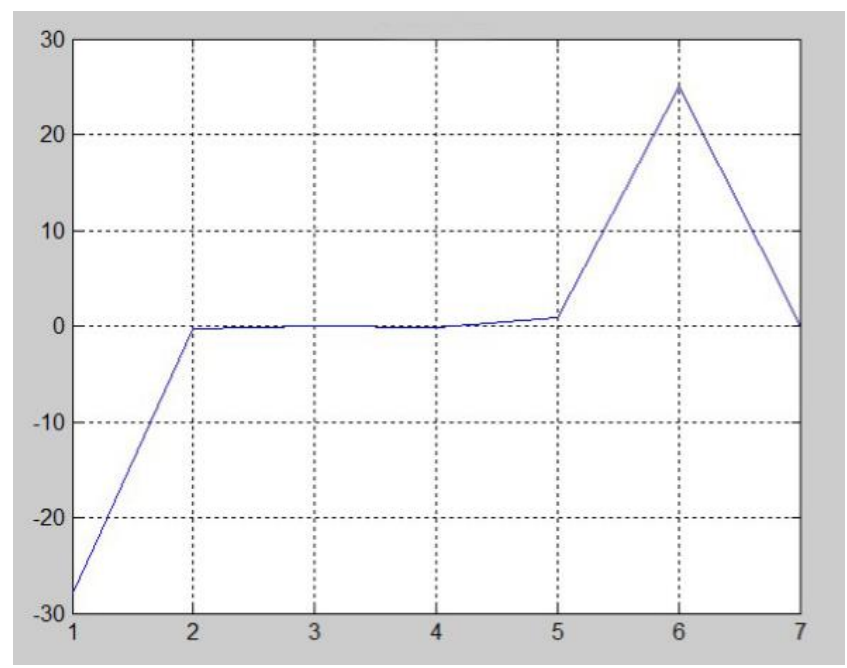

Fig. 4. Network error variation value compared with the real data

TABLE III. ERror CheCKING With BP NeURAL Network Model

\begin{tabular}{|c|c|c|}
\hline $\begin{array}{c}\text { Original Residual } \\
\text { Sequence }\end{array}$ & $\begin{array}{c}\text { BP Neural Networks } \\
\text { Simulation } \\
\text { Prediction }\end{array}$ & Prediction Error \\
\hline-1779.17 & -1751.3 & $1057 \%$ \\
\hline 319.66 & 319.9 & $0.08 \%$ \\
\hline 444.36 & 444.4 & $0.01 \%$ \\
\hline 674.96 & 675.2 & $0.04 \%$ \\
\hline-526.84 & -527.8 & $0.18 \%$ \\
\hline 1277.33 & 1252.4 & $1.95 \%$ \\
\hline
\end{tabular}

Determine the new predicted value of $\mathrm{q}_{\mathrm{c}}^{(0)}(\mathrm{t})$.We predict the next batch residual is $\mathrm{q}_{\mathrm{c}}^{(0)}(\mathrm{t})=-800.8$ with the trained BP neural network above. Predicted value is $\mathrm{x}_{\mathrm{c}}{ }^{(0)}(\mathrm{t})=84.26$ with the grey theory. So the predictive result after the residual correction for BP neural network is

$$
\begin{aligned}
& X_{c}{ }^{(0)}(t)=X_{c}{ }^{(0)}(t)+q_{c}{ }^{(0)}(t)=11139.34+(-800.8) \\
& =10338.54
\end{aligned}
$$

The predicted value of the next batch of fiber-optic gyroscope scale factor is 10338.54 . In fact, the actual data is 10694.20. The error is $3.3 \%$, which is smaller than general requirements $5 \%$. Next we verified the sample of 65 products in the first quarter of 2013, and we found that the average error is around $3.11 \%$. So we verify that the algorithm described in this paper is feasible to forecast the quality of fiber-optic gyroscope.

\section{CONCLUSION}

In this paper, considering the characteristics of fiber-optic gyroscope, we predict the quality with the grey theory method and optimize the results based on BP neural network. The grey theory is adopted as forecast main body and it is combined with BP neural network to carry on the residual error correction. The case study verify the model is feasible. According to the forecast results, we can take timely measures to control the product quality in advanced.

\section{ACKNOWLEDGMENT}

This work is supported by Shanghai economic and Information Commission under Grant No. 201502016. The authors are grateful for the financial support, and also would like to thank the anonymous reviewers and the editor for their comments and suggestions.

\section{REFERENCES}

[1] Zhou, H. (2012). Application and development of fiber optic gyroscope. Technology Foundation of National Defence, 41-50.

[2] Ma, J., Ye, M., Chao, D. H., Chen, S. Y., \&Yu, M. (2012). Reliability estimation of FOGs based on information fusion technology of multi-sources. Journal of Chinese Inertial Technology, 20 (6), 759-764.

[3] Chang, Pei, C., Lin, J. J., \&Dzan, W. Y. (2012). Forecasting of manufacturing cost in mobile phone products by case-based reasoning and artificial neural network models. Journal of Intelligent Manufacturing, 23 (3), 517-531.

[4] Wu, H. H., \&Shieh, J. I. (2006). Using a Markov chain model in quality function deployment to analyse customer requirements. The International Journal of Advanced Manufacturing Technology, 30(1-2), 141-146.

[5] Deng, Z. H., Zhang, X. H., Liu, W. (2009). A hybrid model using genetic algorithm and neural network for process parameters optimization in $\mathrm{NC}$ camshaft grinding. The International Journalof Advanced Manufacturing Technology, 45(9-10), 859-866.

[6] Wang, X. Q., Qi,L., \&Chen, C. (2014). Grey System Theory based prediction for topic trend on Internet. Engineering Applications of Artificial Intelligence, 29, 191-200.

[7] David, K., Leo, P., \&William, R. (1993). The Application of Artificial Neural Networks to Quality Control Charts. System Sciences, 1993,Proceeding of the Twenty-Sixth Hawaii International Conference on, (pp. 616-625). IEEE.

[8] Wang, W. L., Conley, J. G., Yan, Y. N. (2000). Towards intelligent setting of process parameters for layered manufacturing. Journal of Intelligent Manufacturing, 11(1), 65-74. 
[9] Sanjukta, P., \&William, J. K. (1997). Neural Networks and Evolutionary Computation for Real-time Quality Control of Complex Processes. Reliability and Maintainability Symposium. 1997 Proceedings,(pp. 327-332). IEEE

[10] Yao, T. X., Lin, Y., \&Liu, S. F. (2012). Segmental Correction Connotation GM(1,1) Model. Journal of Grey System, 24(4), 317-326.

[11] Wei, Q. Y. (2011). Quality Control of Small-Batch Production Based on the Improved Grey RBF Neural Network Model. Zhengzhou University, 22-53.

[12] Chen, C. Y. (2007). Accumulative generation of improved gray model and GM $(1,1, \mathrm{t})$. Journal of Mathematics Practice and Understanding, 37(2), 105-109.

[13] Wang, F. X. \&Zhang, L. S. (2009). Combination Gray Forecast Model Based on the Ant Colony Algorithm. Mathematics in Practice and Theory, 39(14), 102-106.

[14] Wan, Y. C., He, Y. Q., \&Cheng, Z. H. (2003). Material consumption based on grey system and neural network generalized weighted function average combination forecast model research. Systems Engineering Theory and Practice, 23(7),80-87.

[15] Lin, Husan, L. (2012). The use of the Taguchi method with grey relational analysis and a neural network to optimize a novel GMA welding process. Journal of Intelligent Manufacturing, 23(5), 1671-1680.

[16] Nitin, K. S., Saurav, D., \&Siba, S. M. (2012). Establishing green supplier appraisement platform using grey concepts. Grey Systems: Theory and Application, 2(3), 395-418.
[17] Tang, X. Q. (2012). Error Modeling and Compansating of Fiber Optic Gyro Based on Wavelet Analysis and Grey Neural Network. Chinese Journal of Lasers,39 (10), 1-6.

[18] Zhang, F., Jia, Z. P., \&Xia, H. (2012). Node trust evaluation in mobile ad hoc networks based on multi-dimensional fuzzy and Markov SCGM $(1,1)$ model. COMPUTER COMMUNICATIONS,35(5), 589-596.

[19] Na, Y.L., Li, L. K., Wu, Y. J., Sun, G. F., \&Yu, H. Y. (2012). Measurement error analysis of FOG's scale factor. Journal of Chinese Inertial Technology, 20(4),472-477.

[20] Schloffel, G. (2013). Unidirectional fiber optic sensor for angular acceleration measurement. Optics Letters, 38(9), 1500-1502.

[21] Wang, B. J., \&Song, G.B. (2002). Engineering bidding decision-making method based on fuzzy mathematics. Journal of Hebei University of technology, 31(2), 106-111.

[22] Li, G. D., \&Nagai, M. (2013). An optimal prediction model for time series prediction in manufacturing systems. The International Journal of Advanced Manufacturing Technology, 67(9-12), 2343-2349.

[23] Li, G. D., Masuda, S., Wang, C. H. (2010). The hybrid grey-based model for cumulative curve prediction in manufacturing system. The International Journal of Advanced Manufacturing Technology, 47(1-4), 337-349. 